\title{
Method for Switching between Traction and Brake Control for Speed Profile Optimization in Mountainous Situations
}

\author{
Nan Lin ${ }^{1,2}$, Changfu Zong ${ }^{1}$ and Shuming Shi ${ }^{2, *}$ \\ 1 State Key Laboratory of Automotive Simulation and Control, Jilin University, Jilin 130022, China; \\ linnan@jlu.edu.cn (N.L.); Zong.Changfu@jlu.edu.cn (C.Z.) \\ 2 Transportation College, Jilin University, Jilin 130022, China \\ * Correspondence: shishuming@jlu.edu.cn
}

Received: 16 October 2018; Accepted: 4 November 2018; Published: 6 November 2018

\begin{abstract}
Making full use of front road grade information to achieve the best fuel efficiency is important for intelligent vehicles. Normal theoretical studies pay too much attention to engine continuous feedback control. The theoretical foundation of switching between traction and brake control has been ignored. In mountainous terrain, both the engine and road slopes are energy sources. Switching between traction and brake control is the key point. This research focuses on broadening the normal control range. The comprehensive objective function that contains traction and brake control is built, and then the analytical switching control law is derived based on Pontryagin's maximum principle (PMP). Analytical switching control laws express the mechanism of switching between traction and brake control for economic cruise control (ECC). Simulation results show that the model can solve the switch time and the entire speed profile precisely. Brake control is very important in downhill situations. The parameters in the objective function influence not only the switch time but also the switch process. This research offers a theoretical foundation for ECC with road slopes and can make onboard control more precise and efficient.
\end{abstract}

Keywords: economic cruise control; road slope; switch control; optimal control; heavy duty vehicle

\section{Introduction}

Using information from the surrounding environment is a promising technique for improving the fuel efficiency of road vehicles. Many manufacturers have equipped their products with economic cruise control (ECC) systems. In mountainous highway situations, disturbances from traffic flow are small, while the vertical profile of roads change frequently. This implies that optimizing the speed profile based on road slope has great potential for saving fuel, especially for heavy duty vehicles [1]. During downhill situations, the inertial force from the road slope may act as a traction force instead of a vehicle engine.

There are three main ways to achieve ECC with road slopes: analytical methods, numerical calculations and intelligent algorithms.

Analytical methods based on optimal control theory have advantages in terms of accuracy, real-time performance and mathematical foundation. Pontryagin's maximum principle (PMP) can be used to derive optimal control laws. In the early 1970s, Schwarzkopf [2], from the University of Oklahoma, regarded the best fuel consumption as a cost function and derived the analytical solutions for some simple situations. David [3] (University of Pennsylvania, Philadelphia, PA, USA), Fröberg [4] (Linkoping University, Linköping, Sweden) and He Chaozhe [5] (University of Michigan, Ann Arbor, Michigan) adopted a linear fuel consumption model and concluded that keeping constant speed is the 
best strategy for small road slopes. He Chaozhe systematically derived the bang-bang or bang-arc-bang control laws with PMP. The longitudinal control input switches between traction and slide according to the switching function. When the switching function satisfies the singular arc condition, the controller performs feedback control to maintain a constant speed. However, such a conclusion cannot be widely accepted because of the strong nonlinear property of engines

Many researchers have introduced a nonlinear engine model to express the economic operation range of engines. Engin Ozatay [6], from Ford Motor Company, modelled the objective function with the square of the vehicle speed, while Benjamin Passenberg [7], from the Technical University of Munich, considered the square of the engine torque. In 2017, Shaobing Xu and Shengbo Li [8] established an instantaneous feedback control algorithm based on PMP and kinetic energy conversion. These models can express the economic operation range of engines to some extent. However, nonlinear state equations and discontinuous gear shifts present difficulties in solving analytical control laws. An analytical solution is suitable for describing the total vehicle power consumption from a macroscale perspective but is not suitable for modelling precise engine fuel consumption.

Numerical algorithms are suitable for more complicated and precise vehicle models. Dynamic programming (DP), nonlinear programming (NLP) and model predictive control (MPC) can be applied to solve a formatted model. Professor Nielsen, from Linkoping University, applied numerous numerical algorithms. Many attempts have been made to simplify the engine model $[9,10]$. The nonlinearity of the engine has been analyzed in detail [11]. The most widely used numerical algorithm is DP [12]. MPC is also used to reduce the computation load by transforming global optimization to local optimization [13]. In 2010, Huang [14], from Auburn University, modelled an NLP algorithm for ECC. Such numerical algorithms have good performance when dealing with real road slope information, but their computational load is very heavy.

To reduce the computational load for vehicle online control, intelligent algorithms and experience-based algorithms have been developed by many researchers. Ermin [15] proposed an expert cruise control system that relied on the logical reasoning of experienced drivers. Ganji [16] and Hamid Khayyam [17] used fuzzy logic, trained by the results of the numerical algorithm, to realize ECC. Such technologies are based on engineering experience, which requires theoretical support.

The common disadvantage of these three methods is that they seldom consider brake control. From the perspective of the control range, all of these studies pay too much attention to the continuous control or the feedback control of traction, while brake control has been ignored. Some models consider braking $[7,9]$, but the control laws are not designed based on energy or fuel savings. Although braking does not cause additional fuel consumption, it is important for speed control. When vehicles drive in mountainous situations, downward slopes in addition to vehicle engines offer traction forces. From the perspective of power management, slope energy can only be passively received. This is different from the vehicle engine, which can be actively controlled. Brake control can be regarded as the downward slope energy controller and can balance two different energy sources. When cruising on rolling hills, switching among traction, slide and brake control is very important. When and where to select the appropriate control action still needs to be studied.

Therefore, switching between traction and brake control is investigated in this paper. The objective function contains both the traction and brake control range. This can optimize the traction and brake control in terms of minimum energy consumption. PMP is used to deduce the analytical control laws. The conclusions can increase the understanding of ECC and can also realize online control with higher accuracy and lower computation load. The analytical control laws offer a theoretical foundation for ECC products.

This paper is organized as follows. In Section 2, ECC in rolling hills situations is modelled as the optimal control problem. The objective function with a united form of the traction and brake control range is formulated. PMP is introduced to deduce the optimum control laws in Section 3. Then, the boundary value problem of differential equations is transformed to a nonlinear equation problem. In Section 5, two typical simulation results are carried out. The results show that brake 
control plays an essential role in downward slope control. The effects of the parameters in the energy model are also analyzed.

\section{Modelling of ECC}

\subsection{Equivalent Fuel Consumption Model}

Broadening the control range is the key point when modelling the objective function. Although fuel cost during braking can be ignored, an objective function without brake control leads to an incomplete control range. Traction and brake control are equality important for speed control. To consider brake control in the view of total energy management, a comprehensive objective function is needed.

Equivalent fuel consumption (EFC) can be introduced to express both the traction and brake energy. In the traction condition, the instantaneous fuel consumption of a heavy duty vehicle has a good linear relationship with the vehicle power (Figure 1). In the brake condition, the heat loss from braking should be taken into consideration. Kinetic energy reductions by braking can be regarded as EFC with a certain proportion. To obtain a uniform equation, linear fitting with the absolute value of the control variable is introduced.

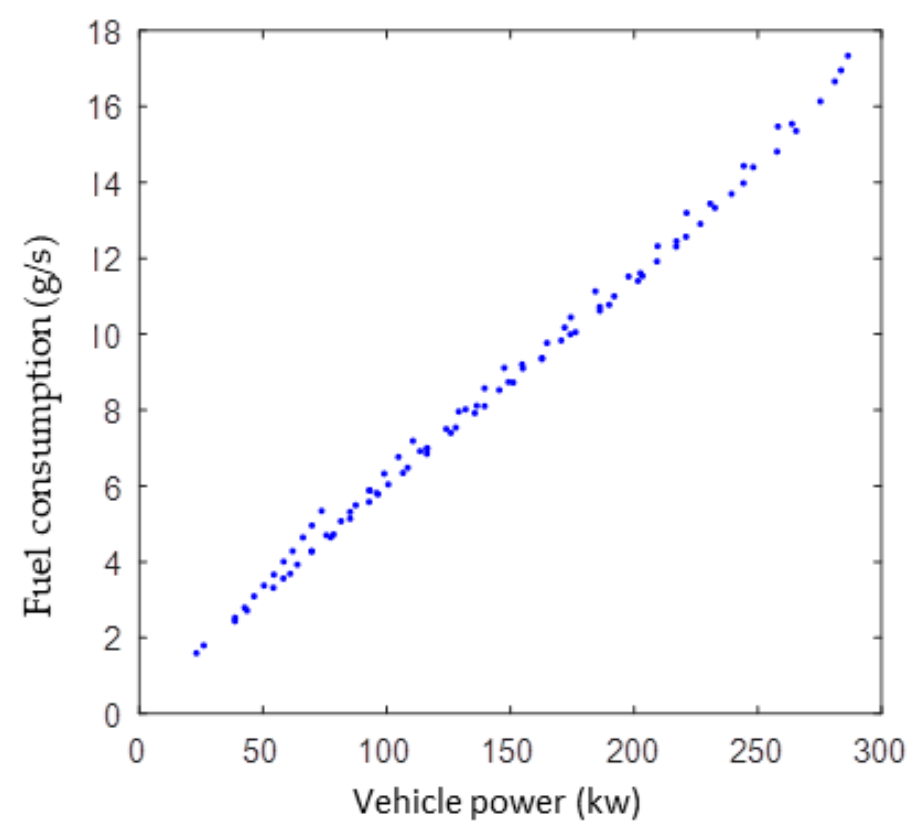

Figure 1. Engine fuel consumption (from the engine bench test).

Shown as Figure 2, EFC can be designed as Equation (1):

$$
\delta=c_{1}\left|F_{l}\right| v+c_{2} F_{l} v
$$

where $c_{1}, c_{2}$ are fitting parameters, and $c_{1} \geq c_{2}>0$. Their value can be solved by the real fuel consumption rate $k^{+}$and designed brake energy transformation rate $k^{-}$. When $c_{1}>c_{2}$, transform the brake energy to fuel consumption with the certain proportion. When $c_{1}=c_{2}$, the brake energy has been ignored, but the control range still contains the brake section. $v$ stands for vehicle speed. The control variable is longitudinal traction or brake force $U=\left[F_{l}\right]$, and $F_{b \max } \leq F_{l} \leq F_{t \max } . F_{t \max }$ is the maximum traction force, and $F_{b \max }$ is the maximum brake force. Because of the gear box, $F_{t \max }$ should be a function of vehicle speed. In order to simplify this problem, and because brake control was always carried out during high-speed cruising, we set a constant value for $F_{t \max }$. The maximum traction force under the smallest gear ratio is selected. 


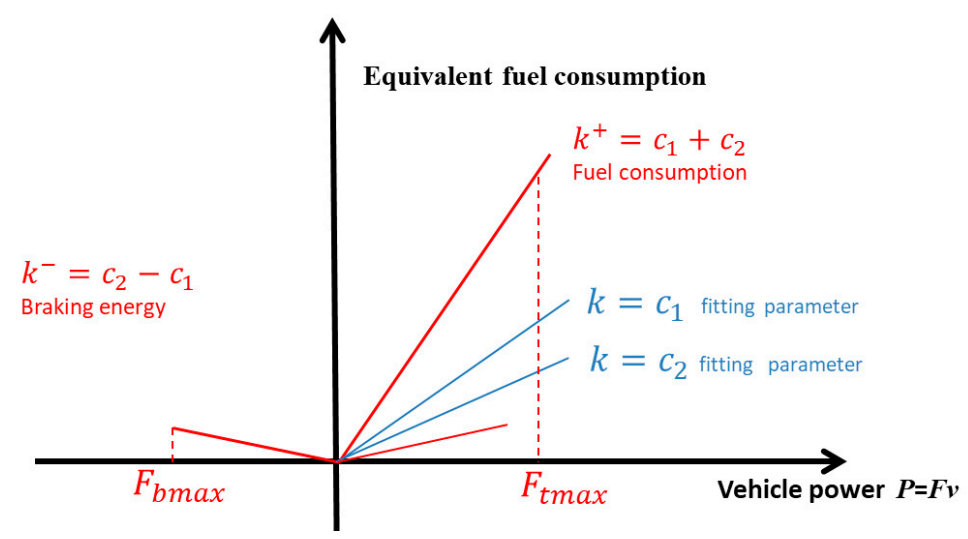

Figure 2. Equivalent fuel consumption (EFC) with both traction and brake control.

In addition, time consumption should also be considered. Here, we use time factor $\sigma$ to design an energy-time optimal control model. The final objective function is illustrated as Equation (2),

$$
J=\int_{0}^{t_{f}}\left(c_{1}\left|F_{l}\right| v+c_{2} F_{l} v+\sigma\right) d t
$$

\subsection{State Equation Based on Longitudinal Dynamics}

The vehicle longitudinal dynamics can be modeled as

$$
F_{l}=F_{f}+F_{w}+F_{i}+F_{j}
$$

The sum of four kinds of resistance is equal to the value of longitudinal control force $F_{l} . F_{f}=m g f$ is rolling resistance, $F_{w}=\frac{1}{2} C_{D} A \rho v^{2}$ is air drag, $F_{i}=m g i$ is gradient resistance and $F_{j}=m \dot{v}$ is acceleration resistance. Other variables include $m$-vehicle mass; $g$-gravitational acceleration; $f$-rolling resistance coefficient; $i$-vehicle acceleration; $C_{D}$ —drag coefficient; $A$-frontal area; $\rho$-air density; $i$ - sine of the road slope angle.

By simplifying the expression of air drag with $k=\frac{1}{2} C_{D} \rho A$, the longitudinal dynamic can be expressed as

$$
F_{l}=m g i+m g f+k v^{2}+m \dot{v}
$$

Here we model the road slope as a linear function of road distance:

$$
i(s)=a s+b
$$

where $s$ is the road distance, $a$ and $b$ are the parameters of the road slope function. This model can represent the concave and convex vertical profile of the road.

Vehicle speed and road distance are selected as state variables. The state vector can be expressed as $X=\left[\begin{array}{c}s \\ v\end{array}\right]$, and the state function is as follows:

$$
X=\left[\begin{array}{c}
\dot{s} \\
\dot{v}
\end{array}\right]=\left[\begin{array}{c}
v \\
\frac{F_{l}}{m}-g(a s+b)-g f-\frac{k v^{2}}{m}
\end{array}\right]
$$

\section{Analytical Solution of Switch Control Based on Optimal Control Theory}

In this section, PMP is applied to derive the optimal control laws and the co-state equations. Then, the optimal control laws are substituted into the extended state equation. Finally, the system solution of bang-bang control or bang-arc-bang control can be obtained analytically. 


\subsection{Derivation of the Optimal Control Law}

Based on PMP, the standard Hamiltonian function is defined as

$$
\begin{aligned}
H & =c_{1}\left|F_{l}\right| v+c_{2} F_{l} v+\sigma+\lambda_{s} v+\lambda_{v}\left(\frac{F_{l}-G i(s)-G f-k v^{2}}{m}\right) \\
& =c_{1} v\left|F_{l}\right|+\left(c_{2} v+\frac{\lambda_{v}}{m}\right) F_{l}+\sigma+\lambda_{s} v+\lambda_{v}\left(-g(a s+b)-g f-\frac{k v^{2}}{m}\right)
\end{aligned}
$$

Since $H$ is a linear function for the control variable $F_{l}$, minimizing the value of $H$ is equivalent to minimizing $R=\left|F_{l}\right|+\left(\frac{m c_{2} v+\lambda_{v}}{m c_{1} v}\right) F_{l}$. The optimal control law is based on $\delta=\frac{m c_{2} v+\lambda_{v}}{m c_{1} v}$, and $\delta$ is called the switch function. The minimum condition gives the controller

$$
\left\{\begin{array}{crr}
F_{l}^{*}(t)=F_{t \max } & \text { if } \delta<-1, \text { or } \lambda_{v}<-m c_{2} v-m c_{1} v \\
0 \leq F_{l}^{*}(t) \leq F_{t \max } & \text { if } \delta=-1, \text { or } \lambda_{v}=-m c_{2} v-m c_{1} v \\
F_{l}^{*}(t)=0 & \text { if }-1<\delta<1, \text { or }-m c_{2} v-m c_{1} v<\lambda_{v}<m c_{1} v-m c_{2} v \\
F_{b \max } \leq F_{l}^{*}(t) \leq 0 & \text { if } \delta=1, & \text { or } \lambda_{v}=m c_{1} v-m c_{2} v \\
F_{l}^{*}(t)=F_{b \max } & \text { if } \delta>1, & \text { or } \lambda_{v}>m c_{1} v-m c_{2} v
\end{array}\right.
$$

When $\delta= \pm 1$, singular arc control dominates. The optimal control on the singular arc can be obtained according to the Legendre-Clebsch condition. Chaoze He solved the singular arc control law under traction conditions (5), that is, during constant speed. During braking, although the same conclusion can be derived, the strict generalized Legendre-Clebsch condition is not satisfied, that is, $-\frac{\partial \ddot{\delta}}{\partial F_{l}}<0$. Thus, the conclusion can be drawn that there is no singular arc on brake control. The final optimal control law is as follows:

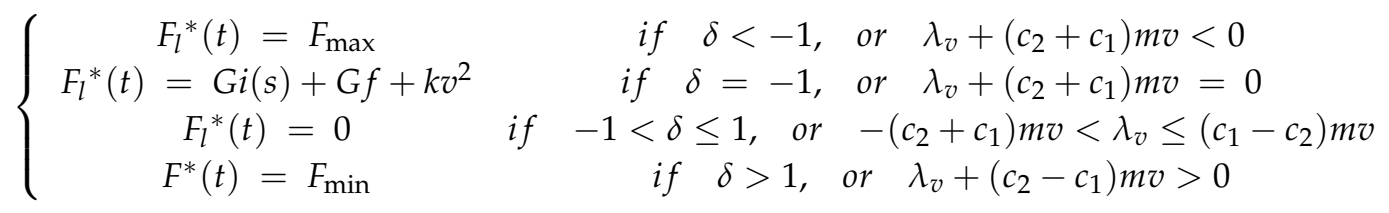

Therefore, ECC with road slopes can be realized by bang-bang control or bang-arc-bang control. It is beneficial to select each control action according to the switch function.

\subsection{The Extended State Equation under the Optimal Control Law}

The co-state equations can be derived from the Hamiltonian function:

$$
\left[\begin{array}{l}
\dot{\lambda}_{s} \\
\dot{\lambda}_{v}
\end{array}\right]=\left[\begin{array}{c}
-\frac{\partial H}{\partial s} \\
-\frac{\partial H}{\partial v}
\end{array}\right]=\left[\begin{array}{c}
\lambda_{v} g a \\
-\lambda_{s}+\frac{2 \lambda_{v} k v}{m}-c_{1}\left|F_{l}\right|-c_{2} F_{l}
\end{array}\right]
$$

According to the optimal control law and the co-state equations, the extended state equation can be illustrated as Equation (11). Here, we simplify the air drag in a linear form, where $\bar{v}$ denotes the average cruising speed.

$$
\left[\begin{array}{c}
\dot{s} \\
\dot{v} \\
\dot{\lambda}_{s} \\
\dot{\lambda}_{v}
\end{array}\right]=\left[\begin{array}{cccc}
0 & 1 & 0 & 0 \\
-g a & \frac{-k \bar{v}}{m} & 0 & 0 \\
0 & 0 & 0 & g a \\
0 & 0 & -1 & \frac{2 k \bar{v}}{m}
\end{array}\right]\left[\begin{array}{c}
s \\
v \\
\lambda_{s} \\
\lambda_{v}
\end{array}\right]+\left[\begin{array}{c}
0 \\
\frac{F_{l}{ }^{*}}{m}-g(f+b) \\
0 \\
-c_{1}\left|F_{l}{ }^{*}\right|-c_{2} F_{l}{ }^{*}
\end{array}\right]
$$

For the normal control process, $F_{l}{ }^{*}$ switches among traction control with maximum traction force, slide control and brake control with maximum brake force. For the singular arc, $F_{l}^{*}(t)=G i(s)+$ 
$G f+k v^{2}=m g(a s+b)+m g f+k \bar{v} v$. Substituting this control law into the extended state equation, we obtain

$$
\left[\begin{array}{c}
\dot{s} \\
\dot{v} \\
\dot{\lambda}_{s} \\
\dot{\lambda}_{v}
\end{array}\right]=\left[\begin{array}{cccc}
0 & 1 & 0 & 0 \\
0 & 0 & 0 & 0 \\
0 & 0 & 0 & g a \\
-\left(c_{1}+c_{2}\right) m g a & -\left(c_{1}+c_{2}\right) k \bar{v} & -1 & \frac{2 k \bar{v}}{m}
\end{array}\right]\left[\begin{array}{c}
s \\
v \\
\lambda_{s} \\
\lambda_{v}
\end{array}\right]+\left[\begin{array}{c}
0 \\
0 \\
0 \\
-\left(c_{1}+c_{2}\right) m g(b+f)
\end{array}\right]
$$

\subsection{Analytical Solution of the Linear State Equation}

After deriving the analytical extended state equation, we then solved specific numerical results. The key point is to solve the switch time and initial value of the co-states. Here, we use a traditional method to solve this boundary value problem.

The discontinuous nature of bang-bang control makes numerical solutions difficult. The traditional shooting method is very sensitive to the initial value and requires a massive calculation. For the proposed simplified linear system, an analytical solution is introduced. First, we assume the control process and derive the system operation with symbolic variables. Then, we solve the equations formulated from the boundary values and switch functions. This transforms the boundary value problem (BVP) to a set of algebraic equations.

The extended state equation describes each variable with a differential equation. The original function can be derived by integration. Therefore, we obtain the solution when the control input is constant:

$$
X(t)=G_{\text {bang }}\left(X_{0}, t_{b}, t_{e}, F_{b a n g}\right), \quad F_{b a n g} \in\left\{F_{b \max }, 0, F_{t \max }\right\}
$$

Here, $X_{0}=\left[\begin{array}{llll}s(0) & v(0) & \lambda_{x}(0) & \lambda_{v}(0)\end{array}\right]^{T}$ is the initial value, and $t_{b}$ and $t_{e}$ are the beginning and ending times, respectively. $F_{b a n g}$ can be chosen from $F_{b \max }, F_{t \max }$ and slide without any traction or brake force.

On the singular arc, the control input can be represented by state variables. According to Equation (12), the function can be represented by

$$
X(t)=G_{\text {arc }}\left(X_{0}, t_{0}, t\right)
$$

The boundary value contains beginning state constraints, ending state constraints and the ending Hamiltonian constraint. All boundary value constraints can be written as follows:

$$
B\left(X(0), X\left(t_{f}\right)\right)=\left[\begin{array}{c}
s(0) \\
s\left(t_{f}\right)-s \\
v(0)-v_{0} \\
v\left(t_{f}\right)-v_{f} \\
H\left(s\left(t_{f}\right), v\left(t_{f}\right), \lambda_{s}\left(t_{f}\right), \lambda_{v}\left(t_{f}\right), F_{l}\left(t_{f}\right)\right)
\end{array}\right]
$$

In addition, the switch function can also be used to form equality contains. Equation (16) is satisfied when switching between traction and slide control:

$$
Z_{p}\left(\lambda_{v}(t), v(t)\right)=\lambda_{v}(t)+m c_{2} v(t)+m c_{1} v(t)
$$

Equation (17) is for switching between brake and slide control:

$$
Z_{n}\left(\lambda_{v}(t), v(t)\right)=\lambda_{v}(t)-m c_{1} v(t)+m c_{2} v(t)
$$


Next, we can either assume or estimate the control process. Although there is no theoretical way to solve this problem, driving experience can offer some clues. Take traction-slide-brake control as an example; the operation of the system can be expressed as

$$
\left\{\begin{array}{l}
X\left(t_{1}\right)=G_{\text {bang }}\left(X\left(t_{0}\right), t_{0}, t_{1}, F_{t \max }\right) \\
X\left(t_{2}\right)=G_{\text {bang }}\left(X\left(t_{1}\right), t_{1}, t_{2}, 0\right) \\
X\left(t_{f}\right)=G_{\text {bang }}\left(X\left(t_{2}\right), t_{2}, t_{f}, F_{b \max }\right)
\end{array}\right.
$$

where $t_{0}=0$ is the beginning time, $t_{1}$ is the time when the control input switches from traction to slide, and $t_{2}$ is the time when the control input switches from slide to brake. After deriving all the symbolic state variables, the simultaneous equations formed by Equations (15), (16) and (17) can be solved. Those unknown variables that contain $\left\{t_{1}, t_{2}, t_{f}, \lambda_{s}(0), \lambda_{v}(0)\right\}$ can be solved. Thus, the entire system can be solved.

\section{Some Typical Simulations}

The simulation results obtained by the proposed method are illustrated in this section. Two typical situations, a convex road profile and a concave road profile, were examined. The simulation parameters are from a heavy duty vehicle and are shown in Table 1.

Table 1. Simulation parameters. EFC: equivalent fuel consumption.

\begin{tabular}{cccc}
\hline & Symbol & Value & Units \\
\hline vehicle mass & $m$ & 48 & tons \\
air drag coefficient & $k$ & 5.3326 & - \\
rolling resistance coefficient & $f$ & 0.0048 & - \\
maximum traction force & $F_{\text {tmax }}$ & 9188 & $\mathrm{~N}$ \\
maximum brake force & $F_{b \max }$ & $-18,376$ & $\mathrm{~N}$ \\
average velocity & $\bar{v}$ & 83 & $\mathrm{~km} / \mathrm{h}$ \\
\hline EFC parameters & $c_{1}$ & $3.88 \times 10^{-5}$ & - \\
(brake energy considered) & $c_{2}$ & $3.00 \times 10^{-5}$ & - \\
\hline EFC parameters & $c_{1}{ }^{\prime}$ & $3.44 \times 10^{-5}$ & - \\
(brake energy unconsidered) & $c_{2}{ }^{\prime}$ & $3.44 \times 10^{-5}$ & - \\
\hline
\end{tabular}

Two sets of EFC parameters are selected for simulation. Their traction fuel consumption is the same, but the brake energy is different. In one set, the brake energy was ignored, but in the other, it was not. The two simulated EFCs are shown in Figure 3.

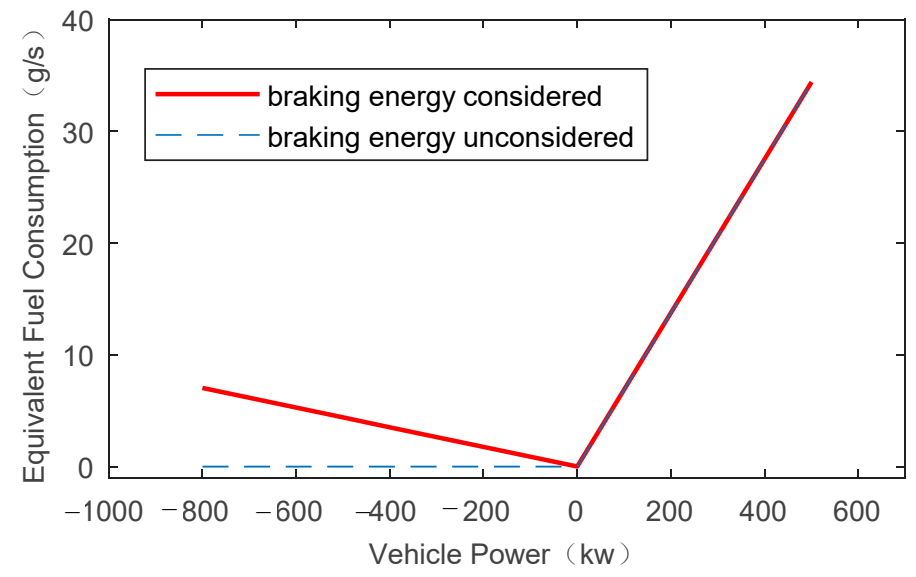

Figure 3. Simulated EFC models. 


\subsection{Convex Road Profile}

The optimization parameters for the convex road profile are shown in Table 2. The road profile and its slope are drawn in Figure 4, showing a small uphill section followed by a long downhill section. The average road slope is $-0.8 \%$.

Table 2. Parameters for the convex road profile situation.

\begin{tabular}{cccc}
\hline & Symbol & Value & Units \\
\hline beginning/ending velocity & $v_{0} / v_{f}$ & 83 & $\mathrm{~km} / \mathrm{h}$ \\
distance & $S$ & 3 & $\mathrm{~km}$ \\
road slope parameter 1 & $a$ & $-1.5 \times 10^{-5}$ & \\
road slope parameter 2 & $b$ & $1.5 \times 10^{-2}$ & \\
\hline
\end{tabular}

(a)

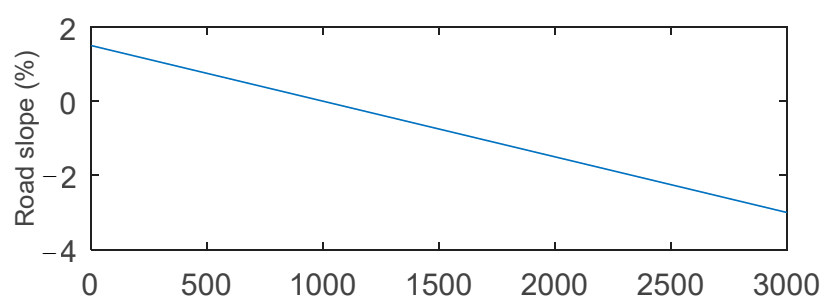

(b)

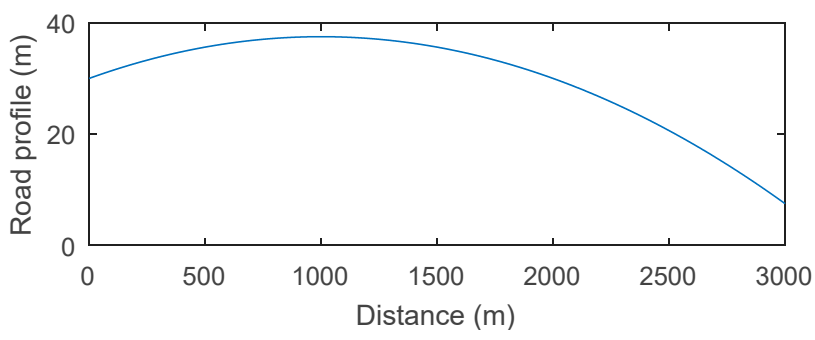

Figure 4. Convex road profile and its road slope. (a) Road slope profile; (b) road profile.

Figures 5-7 illustrate the speed and control input when the time factor is 0,10 and 30. Each group contains a comparison of brake energy considerations. When $\sigma=0$ (Figure 5), only fuel consumption is taken into consideration. The result takes full advantage of road slopes. The switch process is slide-traction. The vehicle slides for a long time, before short-term traction was carried out near the destination. This can help reach the given terminal velocity.

(a)

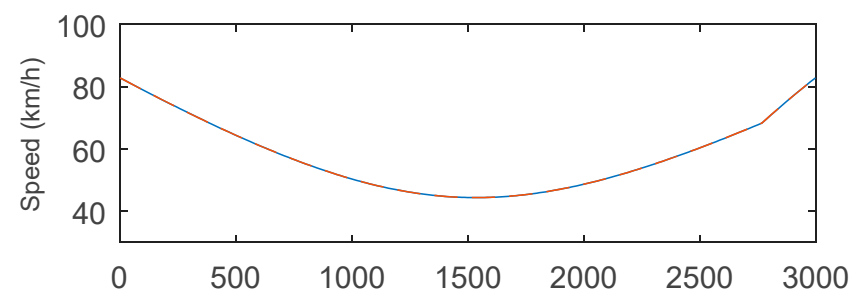

(b)

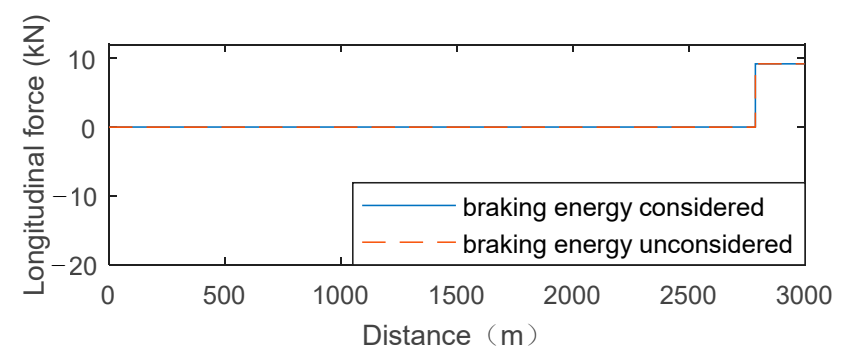

Figure 5. Optimum speed profile and its corresponding control inputs; time factor $\sigma=0$ and duration $t_{f}=194 \mathrm{~s} / 194 \mathrm{~s}$. (a) Speed profile; (b) control input profile. 
(a)

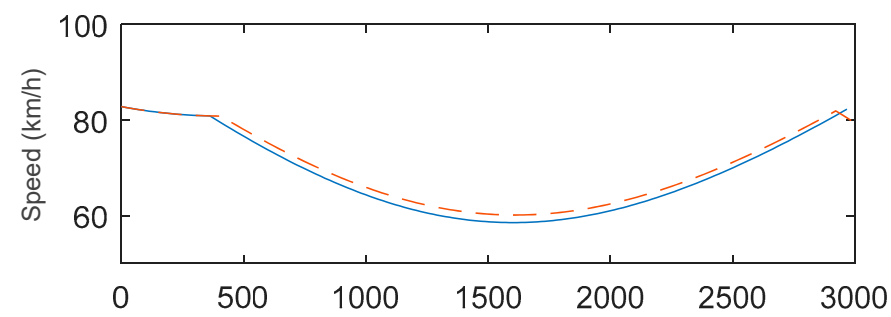

(b)

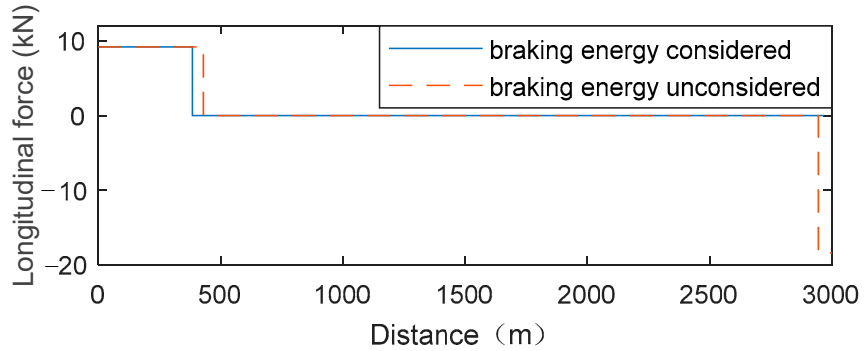

Figure 6. Optimum speed profile and its corresponding control inputs; time factor $\sigma=10$, duration $t_{f}=158 \mathrm{~s} / 155 \mathrm{~s}$. (a) Speed profile; (b) control input profile.

(a)

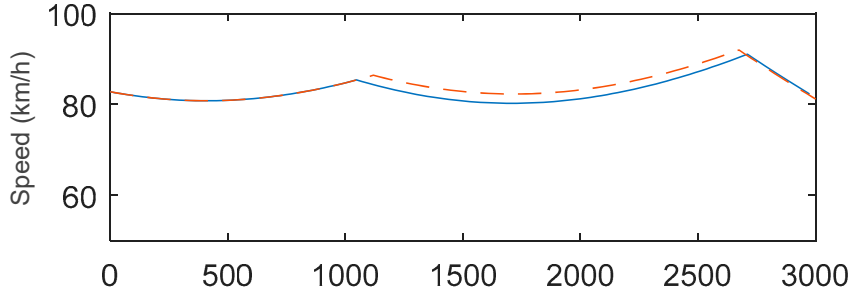

(b)

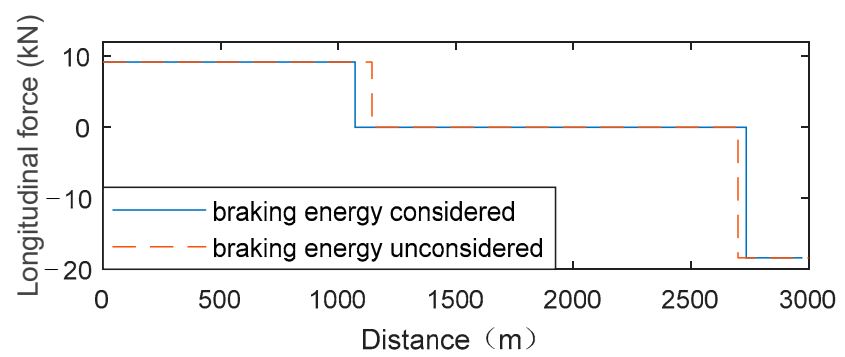

Figure 7. Optimum speed profile and its corresponding control inputs; time factor $\sigma=30$, duration $t_{f}=130 \mathrm{~s} / 128 \mathrm{~s}$. (a) Speed profile; (b) control input profile.

When the time factor increases to 10 (Figure 6), the switch process turns into traction-slide or traction-slide-brake. First, the vehicle maintains a high speed while travelling uphill with traction control. Then, the vehicle slides for a long time. A small brake may be needed near the destination.

The distance of traction and brake control further increases when $\sigma=30$ (Figure 7), while the distance of slide control decreases obviously. The average velocity is higher than the beginning and ending velocity.

Considering that brake energy mainly affects switch time, the average velocity when the brake energy is not considered is always higher than the considered velocity. This can be explained by saving the brake energy for traction control. Thus, ignoring brake energy leads to longer-distance traction control, higher average speed and shorter duration time. In addition, a shorter time also benefits time consumption. In conclusion, ignoring brake energy generates a more aggressive driving style, and the percentage of traction and brake control is relatively high. The value of $c_{1}-c_{2}$ is higher, and the average velocity is lower. This is quite similar to the effects of the time factor. A lower time factor and a higher $c_{1}-c_{2}$ value have similar effects on the speed profile. 


\subsection{Concave Road Profile}

Another set of simulations was carried out on a concave road profile. The parameters are shown in Table 3. The road profile and the slope are drawn in Figure 8, showing a continuous downhill situation that gradually levels off to a flat road. The average road slope is $-1.8 \%$.

Table 3. Parameters for the concave road profile situation.

\begin{tabular}{cccc}
\hline & Symbol & Value & Units \\
\hline beginning/ending velocity & $v_{0} / v_{f}$ & 83 & $\mathrm{~km} / \mathrm{h}$ \\
distance & $S$ & 3 & $\mathrm{~km}$ \\
road slope parameter 1 & $a$ & $7.5 \times 10^{-6}$ & \\
road slope parameter 2 & $b$ & -0.03 & \\
\hline
\end{tabular}

(a)

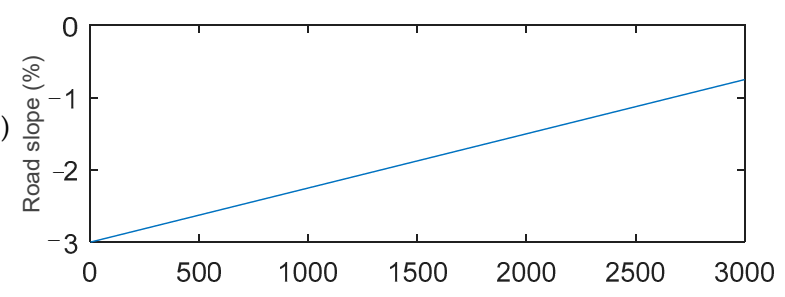

(b)

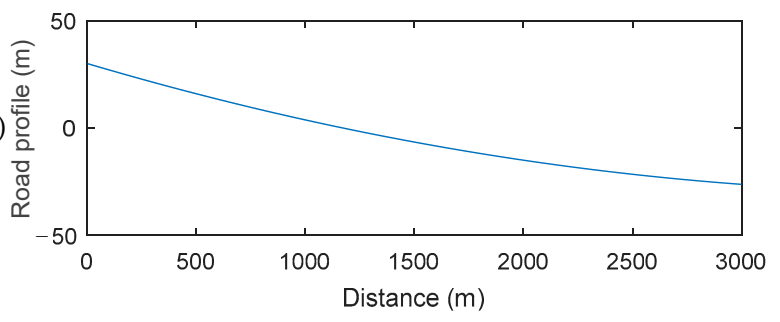

Figure 8. Concave road profile and its road slope. (a) Road slope profile; (b) road profile.

When $\sigma \leq 38$, the optimal solution is unique. As shown in Figure 9, the switch process is slide-brake control. The vehicle accelerates quickly under the condition of downward-sloping hills. Brake control is applied during the last $500 \mathrm{~m}$, and the speed reduces to the ending state constraint. The optimal control law can utilize the road slope as effectively as possible. There is no requirement to use traction control. As high speed can be achieved with low fuel consumption, variation in the time factor does not influence the simulation result. When the time factor is larger than the fuel consumption of traction, additional traction and brake control will be needed. As shown in Figure 10, when $\sigma=40$, the switch process changes to traction-slide-brake control.

Next, we present a quantitative analysis for fuel savings. Cruising at a constant speed is always selected for the control test. Maintaining the same simulation conditions during optimal control and cruising at a constant speed is very important. The main simulation conditions are the duration time and the boundary value. The influence of duration time is obvious. Fuel savings by increasing the duration time are negligible. The boundary value also influences the switch process and the switch time. In fact, it is unrealistic to design a control test with the same duration time and the same boundary value. The constant speed can be calculated by the distance and duration time, but this calculated speed is different from the selected boundary value condition. Therefore, the calculation of the fuel savings percentage is less rigorous. However, due to the steep slope, the simulated situation cannot implement cruising at a constant speed. The limited traction and brake control ranges do not offer sufficient control inputs.

Therefore, only fuel consumption under optimal control is illustrated in Table 4. Since the difference when considering brake energy is small, the result when ignoring brake energy is omitted. Here, RFC denotes real fuel consumption. EFC in terms of the brake energy and time factor is excluded. 

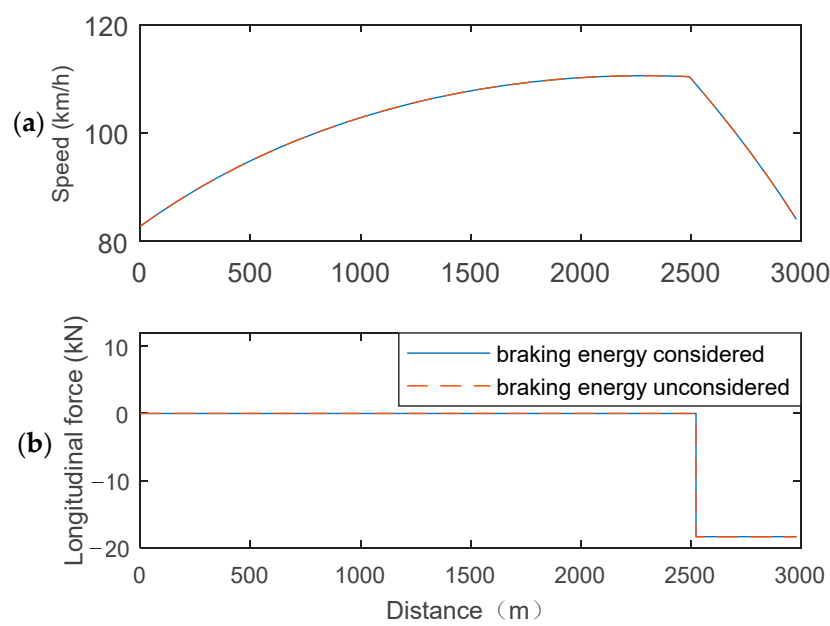

Figure 9. Optimum speed profile and its corresponding control inputs; time factor $\sigma=0 \sim 38$, duration $t_{f}=107 \mathrm{~s} / 107 \mathrm{~s}$. (a) Speed profile; (b) control input profile.

(a)

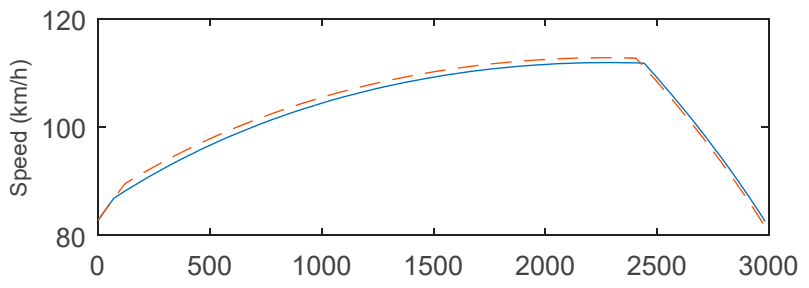

(b)

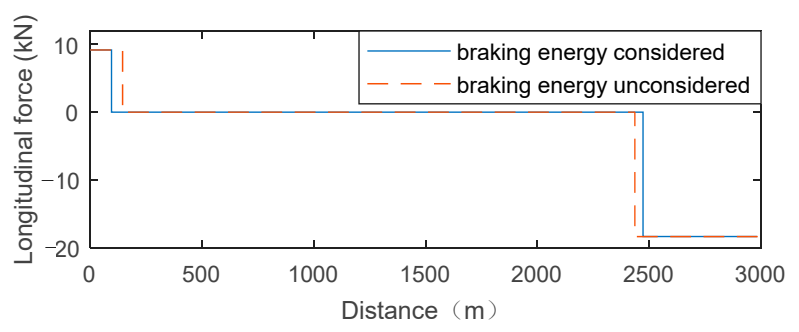

Figure 10. Optimum speed profile and its corresponding control inputs; time factor $\sigma=40$, duration $t_{f}=105 \mathrm{~s} / 104 \mathrm{~s}$. (a) Speed profile; (b) control input profile.

Table 4. The quantitative analysis of the simulated results. RFC: real fuel consumption.

\begin{tabular}{ccccccc}
\hline & Units & \multicolumn{3}{c}{ Convex } & \multicolumn{3}{c}{ Concave } \\
\hline average slope & $\%$ & & -0.8 & \multicolumn{3}{c}{-1.8} \\
\hline time factor & - & 0 & 10 & 30 & $0 \sim 38$ & 40 \\
duration time & & 194 & 158 & 130 & 107 & 105 \\
average speed & $\mathrm{km} / \mathrm{h}$ & 55.7 & 68.4 & 83.1 & 100.9 & 102.9 \\
EFC & $\mathrm{g}$ & 342.1 & 1833.5 & 4619.7 & $78.0 \sim 4144.1$ & 4386.3 \\
RFC & $\mathrm{g}$ & 147.1 & 243.5 & 677.2 & 0 & 59.6 \\
RFC/EFC & $\%$ & 43.0 & 13.3 & 14.7 & 0 & 1.4 \\
\hline
\end{tabular}

From the above table, the fuel savings potential based on the road slope is obvious. Little fuel is needed for the concave situation. The percentage of RFC in the objective function is low, no more than half. The consumption of the time factor is obvious for downhill situations.

\section{Conclusions}

For ECC on rolling hills situations, continuous traction control has been paid too much attention. In mountainous terrain, both the engine and road slopes are energy sources. Switching between traction and brake control is the key point. Downhill slopes require frequent and larger proportions 
of brake control. It is essential to design a comprehensive control method that broadens the general limited control range.

The traction and brake switch control method based on optimal control theory was developed in this paper. A comprehensive objective function that contains traction and brake control was built. Then, the Hamiltonian function was derived with an objective function and other equality constraints. The mathematical framework can be solved analytically based on PMP. The simulation results show that brake control is very important in downhill situations. The parameters in the objective function influence not only the switch time but also the switch process.

This paper focused on analytical control law derivation and illustrated the traction and brake mechanism with typical simulation results. The final numerical solution is only a tentative exploration. Although it can solve this problem with high accuracy and efficiency, its limitation is obvious. The switch process needs to be assumed prior to solving for the switch time. This presents difficulties when the switch process is complicated. Numerical solutions with higher efficiency will be developed in future research.

This research offers a theoretical foundation for ECC with road slopes. Compared with DP and intelligent algorithms, the analytical control law can make onboard control more precise and efficient. Other factors, such as traffic flow and fleet management, can be taken into consideration in the future.

Generally, the proposed model could be integrated into automated vehicles or adaptive cruise control systems to decrease fuel consumption. The proposed model can achieve more obvious fuel savings for rolling hills. For simple road vertical profiles, the control logic is relatively easy and does not have much space for optimization. For rolling hills situations, the frequency of switching between traction and brake control is very high, and the control logic becomes complicated. It is important to balance the energy from both the engine and road slopes. Designing ECC systems with traction and brake active control has obvious fuel benefits and market needs.

Author Contributions: N.L. and S.S. conceived the main concept of the control structure and developed the entire control system. N.L. carried out the research and analyzed the numerical data with guidance from C.Z., N.L. and S.S. collaborated to prepare the manuscript.

Funding: This research was funded by Natural Science Foundation of China $(51805202 ; 51475199)$.

Conflicts of Interest: The authors declare no conflict of interest.

\section{References}

1. Kock, P.; Ordys, A.; Collier, G.; Weller, R. Intelligent Predictive Cruise Control Application Analysis for Commercial Vehicles based on a Commercial Vehicles Usage Study. SAE Int. J. Commer. Veh. 2013, 6, 598-603. [CrossRef]

2. Schwarzkopf, A.; Leipnik, R. Control of highway vehicles for minimum fuel consumption over varying terrain. Transp. Res. 1977, 11, 279-286. [CrossRef]

3. Chang, D.J.; Morlok, E.K. Vehicle speed profiles to minimize work and fuel consumption. J. Transp. Eng. 2005, 131, 173-182. [CrossRef]

4. Fröberg, A.; Hellström, E.; Nielsen, L. Explicit fuel optimal speed profiles for heavy trucks on a set of topographic road profiles. In Proceedings of the SAE World Congress, Detroit, MI, USA, 3-6 April 2006.

5. He, C.; Maurer, H.; Orosz, G. Fuel Consumption Optimization of Heavy-Duty Vehicles with Grade, Wind, and Traffic Information. J. Comput. Nonlinear Dyn. 2016, 11, 061011. [CrossRef]

6. Ozatay, E.; Ozguner, U.; Filev, D. Velocity profile optimization of on road vehicles: Pontryagin's Maximum Principle based approach. Control Eng. Pract. 2017, 61, 244-254. [CrossRef]

7. Passenberg, B.; Kock, P.; Stursberg, O. (Eds.) Combined time and fuel optimal driving of trucks based on a hybrid model. In Proceedings of the 2009 European Control Conference (ECC), Budapest, Hungary, 23-26 August 2009.

8. Xu, S.; Li, S.E.; Cheng, B.; Li, K. Instantaneous Feedback Control for a Fuel-Prioritized Vehicle Cruising System on Highways with a Varying Slope. IEEE Trans. Intell. Transp. Syst. 2017, 18, 1210-1220. [CrossRef] 
9. Fröberg, A.; Nielsen, L. Optimal Control Utilizing Analytical Solutions for Heavy Truck Cruise Control; Linköping University: Linköping, Sweden, 2008.

10. 1Ivarsson, M.; Nielsen, L. Optimal speed on small gradients-consequences of a non-linear fuel map. In Proceedings of the IFAC World Congress, Seoul, Korea, 6-11 July 2008; pp. 3368-3373.

11. Ivarsson, M.; Åslund, J.; Nielsen, L. Look-ahead control-consequences of a non-linear fuel map on truck fuel consumption. Proc. Inst. Mech. Eng. Part D J. Automob. Eng. 2009, 223, 1223-1238. [CrossRef]

12. Hellström, E.; Åslund, J.; Nielsen, L. Design of an efficient algorithm for fuel-optimal look-ahead control. Control Eng. Pract. 2010, 18, 1318-1327. [CrossRef]

13. Hellström, E.; Fröberg, A.; Nielsen, L. A real-time fuel-optimal cruise controller for heavy trucks using road topography information. In Proceedings of the SAE World Congress, Detroit, MI, USA, 3-6 April 2006.

14. Huang, W. Design and Evaluation of a 3D Road Geometry Based Heavy Truck Fuel Optimization System; Auburn University: Auburn, AL, USA, 2010.

15. Kozica, E. Look ahead Cruise Control: Road Slope Estimation and Control Sensitivity; KTH: Stockholm, Sweden, 2005.

16. Ganji, B.; Kouzani, A.Z. Combined quasi-static backward modeling and look-ahead fuzzy control of vehicles. Expert Syst. Appl. 2012, 39, 223-233. [CrossRef]

17. Khayyam, H.; Nahavandi, S.; Davis, S. Adaptive cruise control look-ahead system for energy management of vehicles. Expert Syst. Appl. 2012, 39, 3874-3885. [CrossRef]

(C) 2018 by the authors. Licensee MDPI, Basel, Switzerland. This article is an open access article distributed under the terms and conditions of the Creative Commons Attribution (CC BY) license (http://creativecommons.org/licenses/by/4.0/). 